\title{
Calidad y Aceptabilidad de Chorizos Formulados con Plasma Sanguíneo Bovino y Pasta de Ajonjolí
}

\author{
Lesbia C. Julio, Piedad M. Montero y Diofanor Acevedo* \\ Universidad de Cartagena, Facultad de Ingeniería, Programa de ingeniería de Alimentos, Avenida el \\ Consulado, Calle 30 No. 48-152. Cartagena, Bolívar-Colombia (e-mail: diofanor3000@gmail.com) \\ * autor a quien debe ser dirigida la correspondencia
}

Recibido Mar. 31, 2014; Aceptado Jun. 20, 2014; Versión final recibida Ago. 15, 2014

\begin{abstract}
Resumen
Se evaluó la calidad y aceptabilidad de productos cárnicos tipo chorizo formulados con plasma sanguíneo bovino y pasta de ajonjolí. Para esto el contenido proximal y los análisis microbianos se determinaron siguiendo las Normas Técnicas Colombianas 1663:2009, 1662:2008, 1556:2008 y las normas INVIMA: 1998, respectivamente. El rendimiento se determinó mediante el cálculo de las perdidas por cocción, establecida por la diferencia de peso de las muestras antes y después de la cocción. Para la evaluación de la aceptabilidad se empleó un panel de degustación no entrenado conformado por cincuenta jueces, se utilizó una escala hedónica de cinco puntos. Se observó que es posible reemplazar porcentajes de agua por plasma sanguíneo bovino y tocino por pasta de ajonjolí en la formulación de chorizos. Estos mostraron mejorar el rendimiento, aumentar el contenido proteico y reducir el contenido de grasa, sin que ello tenga un efecto negativo en la aceptabilidad.
\end{abstract}

Palabras clave: plasma sanguíneo, pasta de ajonjolí, productos cárnicos, aceptabilidad

\section{Quality and Acceptability of Chorizos Formulated with Blood Bovine Plasma and Sesame Paste}

\begin{abstract}
The quality and acceptability of sausage type meat products formulated with bovine blood plasma and sesame paste were evaluated. For this the proximal content and microbial analysis were determined following the Colombian Technical Standards 1663:2009, 1662:2008, 1556:2008 and Standards INVIMA: 1998, respectively. The yield was determined by calculating the lost by cooking, established by the difference in weight of the samples before and after cooking. For evaluation of the acceptability a taste panel consisting of fifty untrained judges, a five-point hedonic scale was used. It was observed that it is possible to replace some water by bovine blood plasma and bacon by sesame paste in the formulation of sausages. These showed to improve performance, increase protein content and lower fat content, without having a negative effect on acceptability.
\end{abstract}

Keywords: blood plasma, sesame paste, meat products, acceptability 


\section{INTRODUCCIÓN}

El concepto actual de nutrición está evolucionando, debido a que los consumidores están más atentos al momento de seleccionar los alimentos, ya que ahora no solo es importante, obtener la cantidad de nutrientes suficientes, sino también, la calidad de la ingesta, en términos de nutrientes y no nutrientes, que permite optimizar las funciones fisiológicas de cada individuo para asegurar el máximo de bienestar integral y salud a lo largo de toda su vida (Pacheco et al., 2011). La industria cárnica constantemente evoluciona en la formulación, al utilizar fuentes alternas de bajo costo, capaces de ofrecer alimentos altamente proteicos, con bajo contenido de grasa y con mayor rendimiento y al mismo tiempo conservar las características de calidad deseadas por el consumidor, debido en gran parte a la alta incidencia que ha venido teniendo el consumo de estos productos en el desarrollo de ciertas enfermedades por los altos contenidos de grasa que suelen tener, especialmente de grasa animal. (Vásquez et al., 2010; Pacheco et al., 2011).

Las grasas son fuentes de energía útil para aumentar la densidad calórica de la dieta; sin embargo, es importante cuidar el consumo excesivo de las mismas, ya que se han asociado con varios tipos de enfermedades cardiovasculares y coronarias (De La Torre et al., 2013; Pacheco et al., 2011). De acuerdo con lo anterior, El reto futuro del sector cárnico es establecer alternativas que permitan reducir parcial o totalmente los contenidos de grasa en los productos cárnicos. Dentro de esas alternativas, se destacan el uso de una serie de ingredientes, los cuales pueden contribuir a un mínimo de calorías en las formulaciones y ayudar a mantener las características organolépticas y de proceso que pueden variar por la disminución de la grasa y el aumento de proteína (Pacheco et al., 2011). El ajonjolí tiene un alto valor nutritivo, por la gran cantidad de proteínas, lípidos e importantes minerales y vitaminas. La calidad de su aceite esencialmente con alto contenido de ácido linoleico, hace de esta semilla una alternativa no cárnica para reducir el contenido de grasa animal en productos cárnicos (Acevedo et al., 2013).

En los últimos años se ha presentado un creciente interés en la utilización de plasma sanguíneo obtenido a partir de la centrifugación de la sangre de animales sacrificados, esto se debe a la cantidad y propiedades funcionales de las proteínas sanguíneas (Isaza et al., 2010; Benítez et al., 2011; Rodríguez et al., 2011; Figueroa et al., 2012; Gómez et al., 2013; Julio et al., 2013; Camacho et al., 2014). De igual forma, la proteína sanguínea con calidad microbiológica, debida a la aplicación de adecuados métodos de obtención, tratamientos térmicos y posteriores análisis microbiológicos del plasma (Julio et al., 2011; Benítez et al., 2011; Rodríguez et al., 2011), además de significar una fuente importante de nutrientes, tiene la importancia de reducir los problemas de contaminación ambiental asociados con la descarga de la sangre al medio ambiente por parte de las plantas de beneficio (Figueroa et al., 2012; Julio et al., 2013; Gómez et al., 2013); así mismo, es representativa por el aprovechamiento de un recurso inexplorado en Colombia. En este orden de ideas el objetivo de este trabajo investigativo fue evaluar la calidad nutricional, microbiológica y aceptabilidad de chorizos formulados con plasma sanguíneo bovino y pasta de ajonjolí.

\section{MATERIALES Y MÉTODOS}

\section{Obtención de la sangre y plasma sanguíneo}

La sangre de bovino se obtuvo de la planta de sacrificio del municipio de Arjona-Bolívar; la sangre fue recolectada en recipientes de vidrio de $3 \mathrm{~L}$, se depositaron $12 \mathrm{~g}$ del anticoagulante (citrato de sodio); es decir, $4 \mathrm{~g}$ por cada litro de sangre recolectada. Luego de la sensibilización del animal la sangre fue recolectada directamente de la vena yugular en los recipientes de vidrio. Posteriormente, los frascos con la sangre recolectada se refrigeraron a $4^{\circ} \mathrm{C}$, mediante el uso de neveras con hielo y transportados al laboratorio de Medicina de la Universidad de Cartagena, en donde la sangre se sometió a separación por centrifugación a $3000 \mathrm{rpm}$ durante $10 \mathrm{~min}$ en un equipo marca, KUBOTA, modelo 1200. El plasma fue conservado en refrigeración a $4^{\circ} \mathrm{C}$ (Julio et al., 2011).

\section{Elaboración tecnológica de la pasta de ajonjolí}

El ajonjolí empleado en esta investigación fue obtenido en los Montes de María (Bolívar, Colombia). El primer paso para la elaboración de la pasta es la adecuación de las semillas. Se utilizaron semillas de ajonjolí ( $S$. indicum), de la región de Córdoba Bolívar- Montes de María, mecánicamente descascaradas. Las semillas de sésamo fueron descascaradas como se detalla a continuación: Se tomaron 1000 gramos de semillas, a los cuales se les realizó un proceso de limpieza para eliminar impurezas, luego se sumergieron en 6 litros de agua $\left(T=18 \pm 2^{\circ} \mathrm{C}\right)$ durante $12 \mathrm{~h}$. Las semillas remojadas fueron exprimidas y descascaradas mecánicamente empleando un paño seco para frotar y así desprender la cascara de la semilla. Luego se sumergieron durante cinco minutos en una solución salina al 23\% p/p (cada 1000 gramos en 8 litros de solución), al objeto de separar las cascaras y otros elementos extraños. La inmersión de las semillas en la solución salina, se realizó por triplicado. Las semillas se tomaron desde la superficie de la solución y luego se lavaron con agua cinco (5) veces para eliminar la sal. Posteriormente las semillas se exprimieron para 
reducir el contenido de agua de la superficie de las semillas. Las semillas de $S$. indicum descortezada húmedas fueron utilizadas para el proceso de tostado.

Los gramos de semillas de sésamo descortezada húmedas fueron tostadas a una temperatura controlada en un horno compacto (marca: CHALLERGER, serie 02451) a temperaturas de $130^{\circ} \mathrm{C}$, durante 30 minutos con agitación. Posteriormente, las semillas tostadas fueron molidas en un molino para grano marca corona. Luego la pasta se mezcló con $0,10 \%$ de sal y se homogenizó en un procesador (marca: BLACK \& DECKER), por dos (2) minutos; obteniéndose un producto con consistencia cremosa y sabor definido. Seguidamente, la pasta se envasó en recipientes de vidrio cerrados herméticamente. Se aplicó un tratamiento de pasteurización a $95^{\circ} \mathrm{C}$ por 10 minutos, contados a partir de que el agua comenzó a hervir. Por último, los frascos con la pasta fueron almacenados a $4^{\circ} \mathrm{C}$ (Acevedo et al., 2013)

\section{Formulación y preparación de los tratamientos}

El diseño a utilizar fue de tipo experimental, se manipularon dos variables independientes y se analizó el efecto sobre las variables dependientes. Se desarrolló un diseño completamente al azar, con tres repeticiones. Las variables independientes fueron la incorporación de plasma sanguíneo de bovino y pasta de ajonjolí. Se evaluaron las pérdidas por cocción, calidad microbiológica, aceptabilidad, el contenido de humedad, proteína y grasa del producto. Se definieron los siguientes tratamientos para la elaboración de chorizo: T1 = control. Sin incorporación de plasma sanguíneo (PS) y pasta de ajonjolí (A); T2 = 25\% PS $10 \%$ A; T3 $=25 \%$ PS $-20 \%$ A; T4 $=50 \%$ PS $-10 \%$ A; T5 = 50\% PS $-20 \%$ A. Se utilizó el programa Graph Padlnstats versión 3.1 para aplicar un análisis de varianza (ANOVA), y así, realizar el análisis y comparación de medias, después de haber rechazado la Hipótesis nula de igualdad de medias mediante la técnica ANOVA, se elaboró una comparación de las medias mediante un test de HSD Tukey. El nivel de significancia fue $p \leq 0,05$. También, se empleó el programa Minitab 16 Statistical Software para realizar gráficos de caja y gráficos de medias para intervalos HSD de Tukey.

La formulación de los diferentes tratamientos se presenta en la Tabla 1. Para la preparación de los tratamientos las carnes se pasaron por un molino JAVAR M-12, al igual que el tocino graso. Seguidamente las carnes, la grasa o pasta de ajonjolí y los demás ingredientes con el agua o plasma sanguíneo fueron mezclados hasta obtener una masa homogénea. Los aditivos, el agua o plasma sanguíneo y la sal, se mezclaron perfectamente. Posteriormente, la pasta fue embutida en tripas naturales, se usó una embutidora eléctrica SF 260 RAPID SAUSAGE FILLER, y se amaró manualmente. Los chorizos formados se procesaron térmicamente en un tanque abierto a la atmósfera con agua a $80^{\circ} \mathrm{C}$, calentado en una estufa industrial, hasta que alcanzaron una temperatura interna de $72{ }^{\circ} \mathrm{C}$. Los chorizos cocidos se enfriaron a temperatura ambiente durante $4 \mathrm{~h}$, se pesaron y empacaron al vacío (empacadora JAVAR DZ400) en bolsas de polietileno de alta densidad (Alico® S.A.). Finalmente, fueron almacenados en refrigeración (0-2 ${ }^{\circ} \mathrm{C}$ ) hasta su evaluación.

Tabla 1: Formulación de ingredientes utilizados para los diferentes tratamientos.

\begin{tabular}{|c|c|c|c|c|c|}
\hline \multirow{2}{*}{ Ingredientes (\%) } & \multicolumn{5}{|c|}{ Tratamiontnc } \\
\cline { 2 - 6 } & T1 & T2 & T3 & T1 & T5 \\
\hline Carne de Cerdo & 34 & 34 & 34 & 34 & 34 \\
\hline Carne de res & 34 & 34 & 34 & 34 & 34 \\
\hline Saborizante Chorizo & 1.64 & 1.64 & 1.64 & 1.64 & 1.64 \\
\hline Tocinode cerdo & 13 & 11,7 & 10,4 & 11,7 & 10,4 \\
\hline Pastade aioniolí & - & 1.3 & 2.6 & 1.3 & 2.6 \\
\hline Humo líquido & 0.22 & 0.22 & 0.22 & 0.22 & 0.22 \\
\hline Sal & 0.30 & 0.30 & 0.30 & 0.30 & 0.30 \\
\hline Cebolla puerro & 0.07 & 0.07 & 0.07 & 0.07 & 0.07 \\
\hline Cave & 4.50 & 4.50 & 4.50 & 4.50 & 4.50 \\
\hline Achote & 0.19 & 0.19 & 0.19 & 0.19 & 0.19 \\
\hline Sal curante & 0.26 & 0.26 & 0.26 & 0.26 & 0.26 \\
\hline Aqua & 10.90 & 8.175 & 8.175 & 5.45 & 5.45 \\
\hline Plasma Sanquíneo & - & 2.725 & 2.725 & 5.45 & 5.45 \\
\hline Mezcla Total & 100 & 100 & 100 & 100 & 100 \\
\hline
\end{tabular}




\section{Análisis de proteína, grasa y microbiológicos}

Los contenidos de humedad, materia grasa, proteína y recuento total aerobios, Coliformes totales y fecales se realizaron de acuerdo a lo indicado en las Normas Técnicas Colombianas (NTC) 1663:2009, 1662:2008, 1556:2008 y las normas INVIMA: 1998, respectivamente. Estos últimos se expresaron como Log de UFC/g.

\section{Rendimiento}

Las pérdidas por cocción en los chorizos, se determinó mediante el cálculo de la diferencia de peso de las muestras antes y después de la cocción. Para ello el peso del chorizo antes de la cocción se le restó el peso después de la cocción; a continuación este valor resultante, perdidas por cocción, se restó a un 100\% y de esta manera se obtuvo el rendimiento del producto (Pacheco et al., 2011).

\section{Aceptabilidad}

Para evaluar la aceptabilidad de los productos se empleó un panel de degustación no entrenado, constituido por 50 jóvenes universitarios de ambos sexos, los cuales fueron seleccionados al azar de la Universidad de Cartagena, Colombia. Se utilizó una escala hedónica de 5 puntos como se presenta en la Tabla 2, y se midió el grado de satisfacción que produce cada muestra al ser degustada por los panelistas, determinando así, el grado de aceptabilidad de cada formulación. Se consideró aceptable si la respuesta es "Me gusta mucho" o "Me gusta ligeramente", El análisis de los datos se evaluó por el método Análisis de Varianza (ANOVA); los valores promedios de 1 a 3 se consideran "desagrado", en la escala de 5 categorías; valores superiores corresponden a agrado para los productos evaluados. (Benítez at al., 2008). Para conocer la variabilidad de los datos en la aceptabilidad de los chorizos, se emplearon gráficas de caja y bigotes, estas se obtuvieron utilizando el paquete estadístico Minitab 16 Statistical Software.

Tabla 2: Escala hedónica para evaluar la aceptabilidad del producto

\begin{tabular}{|l|c|}
\hline Escala verbal & Puntuación \\
\hline Me gusta mucho & 5 \\
\hline Me gusta ligeramente & 4 \\
\hline Ni me gusta ni me disgusta & 3 \\
\hline Me disgusta ligeramente & 2 \\
\hline Me disgusta mucho & 1 \\
\hline
\end{tabular}

\section{RESULTADOS Y DISCUSIÓN}

\section{Análisis microbiológico}

La Tabla 3, muestra los resultados de los análisis microbiológicos realizados a los productos y al plasma sanguíneo de bovino. Los resultados indican que el plasma sanguíneo empleado y los chorizos se encuentran entre los parámetros que identifican un nivel de buena calidad para los productos cárnicos procesados cocidos según las normas INVIMA (1998) y la NTC 1325 (2008). Se observó ausencia de coliformes fecales. Aunque la sangre de bovino constituye un caldo de cultivo excelente para los microorganismos ( Barboza et al., 2005) y el excesivo manejo que se genera durante el procesamiento del chorizo pudiera ser fuente de contaminación bacteriana, los valores obtenidos para Aerobios Mesófilos, $<10 U F C / g$ y Coliformes Totales y Fecales, $<3,0 \mathrm{NMP} / g$ se explican debido a que los productos fueron cocidos a $85^{\circ} \mathrm{C}$, hasta alcanzar una temperatura interna de $70^{\circ} \mathrm{C}$, temperaturas a las cuales los microorganismos antes mencionados son altamente sensibles, (Benítez et al.,2011).

Tabla 3: Calidad microbiológica de plasma y chorizos.

\begin{tabular}{|c|c|c|c|c|c|c|}
\hline \multirow{2}{*}{ Conteo } & \multicolumn{6}{|c|}{ Tratamientos } \\
\cline { 2 - 7 } & $\begin{array}{c}\text { Plasma } \\
\text { Sanguíneo }\end{array}$ & $\begin{array}{c}\text { T1 } \\
\text { (Control) }\end{array}$ & $\begin{array}{c}\text { T2 (25\%PS- } \\
10 \% A)\end{array}$ & $\begin{array}{c}\text { T3 (25\%PS- } \\
20 \% A)\end{array}$ & $\begin{array}{c}\text { T4 (50\%PS- } \\
10 \% A)\end{array}$ & $\begin{array}{c}\text { T5 (50\%PS- } \\
20 \% A)\end{array}$ \\
\hline $\begin{array}{c}\text { Mesofilos totales } \\
\text { (UFC/g) }\end{array}$ & $<10$ & $<10$ & $<10$ & 10 & 40 & $<10$ \\
\hline $\begin{array}{c}\text { Coliformes totales } \\
\text { (NPM/g) }\end{array}$ & $<3$ & $<3$ & $<3$ & $<3$ & $<3$ & $<3$ \\
\hline $\begin{array}{c}\text { Coliformes fecales } \\
\text { (NPM/g) }\end{array}$ & $<3$ & $<3$ & $<3$ & $<3$ & $<3$ & $<3$ \\
\hline
\end{tabular}


Los resultados obtenidos en esta investigación referente a la calidad microbiológica de los productos concuerdan con los obtenidos por Isaza et al., (2010) quienes, al realizar 5 productos cárnicos tipo salchichón con diferentes niveles de incorporación de plasma sanguíneo de bovino hidratado, encontraron valores para los análisis microbiológicos dentro de los parámetros aceptados, a excepción de los productos formulados con el $20 \%$ de plasma de bovino hidratado y el control, se atribuyó este resultado al excesivo manejo de estos productos después de su elaboración.

\section{Rendimiento, Contenido de humedad, proteína y grasa.}

Los resultados muestran que hubo diferencia significativa en el rendimiento, Tabla 4. Los productos con plasma sanguíneo y pasta de ajonjolí en su formulación resultaron con un mayor rendimiento $(\mathrm{p} \leq 0,05)$, siendo el tratamiento control y T2 (25\% PS - $10 \%)$, este último con menor contenido de plasma sanguíneo y pasta de ajonjolí, los que presentaron menor rendimiento. El aumento en el rendimiento y la menor pedida de agua en T3 (25\% PS - $20 \%$ A), T4 (50\% PS - $10 \%$ A) y T5 (50\% PS - $20 \%)$ puede ser explicada con base a la propiedad gelificante del plasma, esto se debe a que el gel que se forma atrapa grasa y agua (Barboza et al., 2005), y al contenido de fibra que aporta la pasta de ajonjolí (4,58\%), la cual se hidrató en el proceso de elaboración (Acevedo et al., 2013), ayudando a la retención de agua y disminuyendo las perdidas por cocción. De lo anterior se puede inferir que la incorporación de estos dos componentes mejoró las propiedades de los chorizos. Se reportaron resultados similares en el estudio de Hernández y Güemes (2010), donde la adición de harina de cascaras de naranja redujo las pérdidas por cocción. Este comportamiento también se observó en la investigación realizada por Isaza et al., (2010), la cual mostró que a medida que aumenta el nivel de incorporación de plasma en el producto cárnico, este presenta un mejor porcentaje de rendimiento.

Tabla 4: Rendimiento, Contenido de humedad, proteína y grasa (\%) de los chorizos analizados

\begin{tabular}{|c|c|c|c|c|c|}
\hline Componente & T1 & T2 & T3 & T4 & T5 \\
\hline Rendimiento & $84,57 \mp 1,84^{\mathrm{c}}$ & $87,63 \mp 0,43^{\mathrm{b}}$ & $94,16 \mp 0,42^{\mathrm{a}}$ & $94,35 \mp 0,45^{\mathrm{a}}$ & $95,64 \mp 0,55^{\mathrm{a}}$ \\
\hline Humedad & $65,71 \mp 2,16$ & $63,07 \mp 0,43$ & $63,30 \mp 0,62$ & $63,88 \mp 0,22$ & $66,36 \mp 0,08$ \\
\hline Proteínas & $16,64 \mp 0,51^{\mathrm{c}}$ & $19,26 \mp 0,60^{\mathrm{b}}$ & $22,23 \mp 1,17^{\mathrm{a}}$ & $22,62 \mp 0,06^{\mathrm{a}}$ & $24,17 \mp 0,99^{\mathrm{a}}$ \\
\hline Grasas & $10,18 \mp 0,35^{\mathrm{a}}$ & $9,87 \mp 0,20^{\mathrm{a}}$ & $9,06 \mp 0,18^{\mathrm{b}}$ & $8,92 \mp 0,16^{\mathrm{bc}}$ & $8,40 \mp 0,15^{\mathrm{c}}$ \\
\hline
\end{tabular}

La humedad es otro parámetro medido en los productos cárnicos, Tabla 4. Los chorizos no mostraron diferencia significativa. Este comportamiento se debe a que al incorporar soluciones con un mayor porcentaje de proteína plasmática, aumenta la proporción de materia seca en el producto final, manteniendo un equilibrio entre humedad y materia seca en todos los tratamientos (Rodas et al., 1998). Estudios donde adicionaron de un 5 a $20 \%$ de plasma sanguíneo de bovino en la formulación de productos cárnicos, señalan un ligero aumento en el contenido de humedad con respecto al control y los tratamientos con diferentes niveles de proteína sanguínea (Viana et al., 2005; Isaza et al., 2010).

La Tabla 4, muestra los valores promedios de contenido proteico en los chorizos. Los resultados indican que el agregado de plasma y pasta de ajonjolí, producen diferencias $(p \leq 0,05)$ en el contenido proteico de los productos. Los tratamientos que presentaron mayor porcentaje de proteína fueron T3, T4 y T5. Viana et al., (2005), indican que la incorporación de proteínas de la sangre tanto plasmáticas como globina, permiten obtener un paté de jamón con mayor contenido proteico y reducir de $25-30 \%$ de grasa, con lo que se da la posibilidad de obtener productos cárnicos light, que en general, mantienen el aroma, sabor y consistencia de los productos no modificados. Göket al., (2011) al evaluar el efecto de la utilización de semilla de amapola como sustitutos de grasa en la formulación de hamburguesa, obtuvieron porcentajes altos del contenido proteico para los productos con semilla de amapola en su formulación. Isaza et al., (2010), al remplazar el 10 y $20 \%$ de la proteína de pollo por plasma bovino deshidratado e hidratado encontraron diferencia significativa entre el contenido proteico de los tratamientos.

En cuanto al análisis de grasa el rango de valores va de $8,40 \mp 0,15$ a $10,18 \mp 0,35$, presentando diferencia significativa $(p \leq 0,05)$, Tabla 4 . Los valores más bajos de grasa los presentó el chorizo con mayor contenido de pasta de ajonjolí y plasma sanguíneo en su formulación. Vásquez et al., (2010), estudiaron el efecto de la incorporación de Inulina y Oligofructosa sobre salchichas tipo Viena. Concluyeron que el porcentaje de grasa fue la principal consecuencia de la cantidad de fibra adicionada, es decir, a medida que incrementó la concentración de fibra disminuyó el porcentaje de grasa. Esto mismo, se observó en un estudio realizado por García et al., (2012), quienes elaboraron un producto cárnico tipo hamburguesa con inclusión de harina de quinchoncho (Cajanus cajan), observaron que la adición de harina de quinchoncho afecto significativamente el contenido de grasa en las hamburguesas, ya que, a mayor adición de harina de C. cajan menor contenido de grasa presento el producto final. 


\section{Aceptabilidad}

La aceptabilidad de los chorizos se presenta en la Figura 1. Los datos indican que todos los tratamientos presentan mayor variabilidad en sus datos, a excepción de T5 que presenta similitud en las respuestas de los jueces. Sin embargo, esto no indica cuál de los dos tratamientos es el mejor calificado, solo revela una tendencia de los datos. Por ello se determinó las medias para cada producto, las cuales se muestran en la Figura 2. La misma, consiste en un gráfico de medias para intervalos HSD de Tukey para los dos productos.

Las medias de la prueba de aceptabilidad para T1, T2, T3, T4 y T5 fueron 4,02 $\pm 0,93 ; 3,84 \pm 1,05 ; 3,8 \pm 0,92$; $3,8 \pm 1,14$ y $3,72 \pm 1,07$, respectivamente. Se observó que no hubo diferencias significativas $(p<0.05)$ en los juicios emitidos por los panelistas para los 5 tipos de chorizos evaluados. Se consideró aceptable si la respuesta es "Me gusta mucho" o "Me gusta ligeramente" (Benítez et al., 2008), ver Tabla 2. De acuerdo a lo anterior, se fijó como patrón de aceptabilidad una puntuación igual o superior a cuatro. El control tuvo un porcentaje de aceptación de $70 \%$, seguido de T2 (25\% PS - $10 \%$ ) y T5 (50\% PS - $20 \%$ ) que obtuvieron un $64 \%$ de aceptación, como muestra la Figura 3. De lo anterior se puede decir que es posible incorporar plasma sanguíneo de bovino y pasta de ajonjolí en la formulación de chorizos sin que ello tenga un efecto negativo en la aceptabilidad.

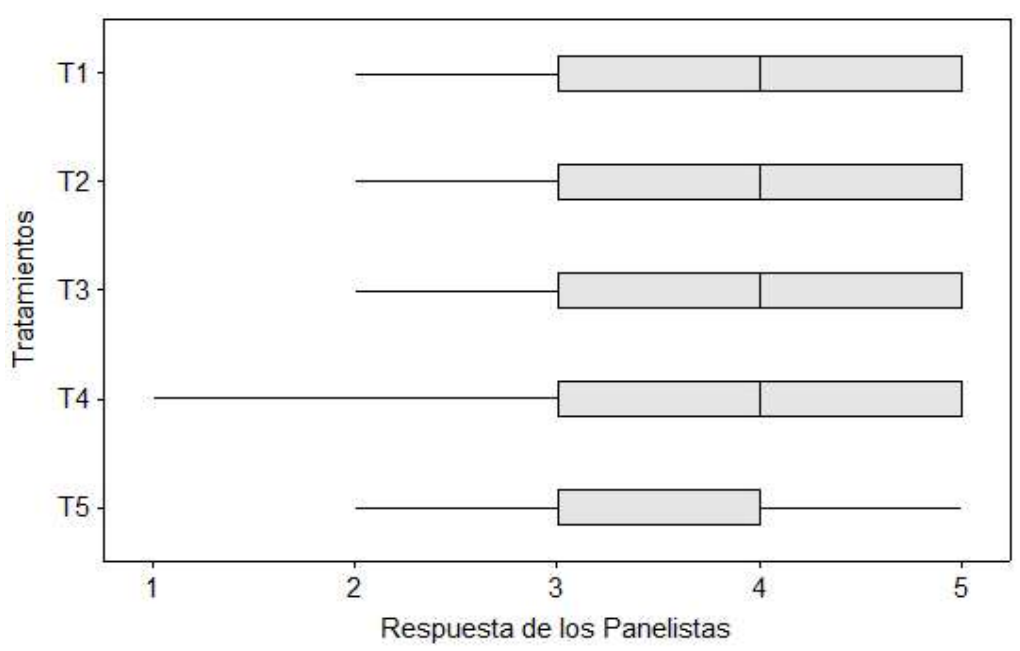

Fig. 1: Variabilidad de las calificaciones asignadas por los panelistas a los chorizos.

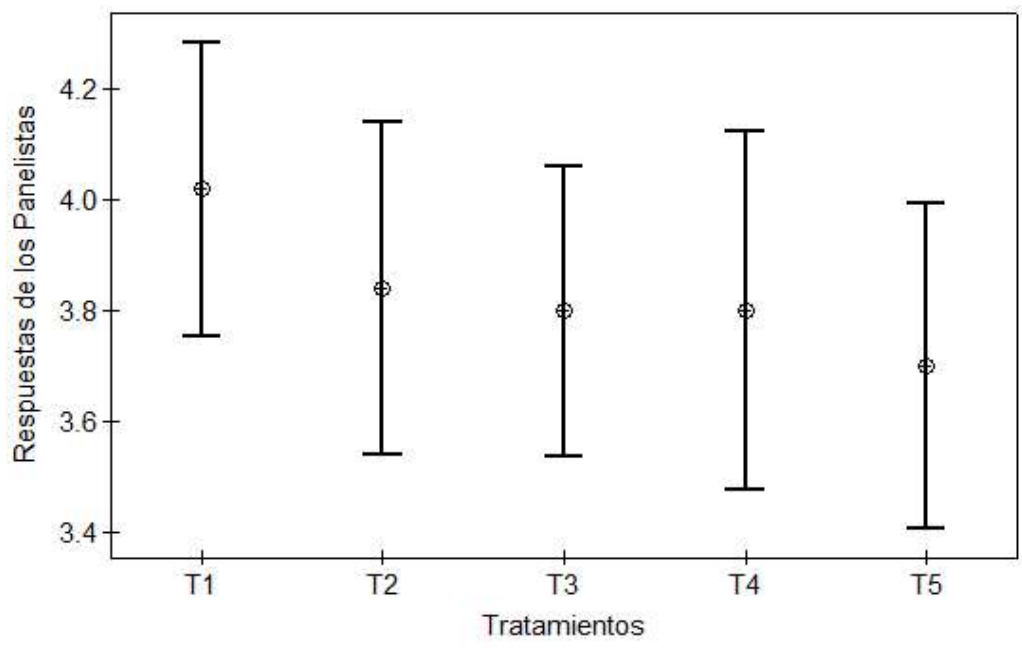

Fig. 2: Medias para intervalos HSD de Tukey para chorizos. 


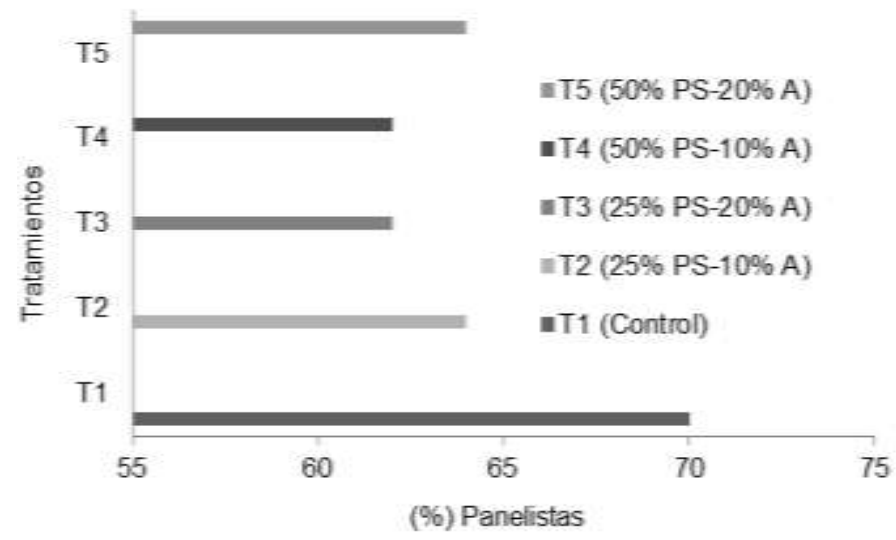

Fig. 3: Porcentaje de panelistas que calificaron los chorizos con puntajes igual o superior a cuatro

Los resultados obtenidos en esta investigación, coinciden con los de Isaza et al., (2010), quienes elaboraron un producto cárnico, tipo salchichón, con incorporación de diferentes concentraciones de plasma sanguíneo y concluyeron que los productos con adición de plasma presentaron buena aceptación por medio de los panelistas. Manchimba y Pambaquishpe, (2007) reportaron que la sustitución de tocino de cerdo por pasta de maní y la incorporación de plasma deshidratado en la elaboración de salchicha tipo Frankfurt, producen diferencias $(p \leq 0,05)$; lo que se concluye que la utilización de Pasta de Maní y Plasma influyen en las características organolépticas del producto; donde el tratamiento T1 es el más aceptable, el mismo que está constituido por $10 \%$ de pasta de maní y $1 \%$ de plasma deshidrato, ya que alcanzó el mayor puntaje otorgado por el panel de degustadores. Por otra parte, Viana et al., (2005) no detectaron diferencias organolépticas sustanciales en paté de jamón con adicionado de plasma sanguíneo de bovino y concluyeron que, en general, la sustitución de la grasa por globina y/o plasma fue beneficioso para la calidad de paté de jamón.

\section{CONCLUSIONES}

Los chorizos y el plasma sanguíneo de bovino presentaron adecuada calidad microbiológica. Lo anterior permite la incorporación de este subproducto de la sangre, en la industria alimentaria. Además de poder ser utilizado para la producción de alimentos funcionales y/o enriquecidos con alto contenido proteico, también se puede decir que es posible remplazar porcentajes de agua por plasma sanguíneo de bovino y tocino por pasta de ajonjolí en la formulación de chorizos, para mejorar el rendimiento, aumentar el contenido proteico y reducir el contenido de grasa, sin que ello tenga un efecto negativo en la aceptabilidad.

\section{AGRADECIMIENTOS}

A la Universidad de Cartagena, a COLCIENCIAS que por medio del Programa de Jóvenes Investigadores e Innovadores 2012-2013, financió el presente trabajo y al Matadero de Arjona - Bolívar.

\section{REFERENCIAS}

Acevedo, D., Y. Marrugo y P. Montero, Evaluación de las propiedades reológicas de pastas de ajonjolí artesanal y tecnificada, Rev. U.D.C.A Act \& Div. Cient.: 16(1), 245-251 (2013)

Barboza, Y., y otros cinco autores, Efecto de la incorporación de proteína plasmática sobre la composición química y calidad proteica de un producto formulado con maíz tierno, Rev. Cient. Fac. Ciencias Vet. U. Zulia: 15(6), 536 - 542 (2005)

Benítez, B. y otros seis autores, Calidad microbiológica de una galleta formulada a base de harina de yuca y plasma de bovino, Rev. Fac. Agron. U. Zulia: 28(2), 260-272 (2011)

Benítez, B., y otros seis autores, Composición proximal, evaluación microbiológica y sensorial de una galleta formulada a base de harina de yuca y plasma de bovino, Interciencia: 33(1), 61-65 (2008)

Camacho, A., P. Montero y M. Duran, Efecto de la adición de plasma sanguíneo de bovino en el contenido proteico, aceptabilidad y calidad microbiológica de una bebida a base de arroz, Revista Alimentos Hoy: 22(31), 104-108 (2014) 
De La Torre, M. S., y otros tres autores, Grasa bovina con un perfil de ácidos grasos mejorado: bajo contenido de grasa saturadas y alto de CLA cis9, trans11, Avances en la calidad de carne bovina: 58, 60-63 (2013)

Figueroa, O., J. Zapata y G. Gutiérrez, Modelamiento de la cinética de hidrólisis enzimática de proteínas del plasma bovino, Revista EIA: 9(17), 71-84 (2012)

García, O., J. Ruíz y I. Acevedo, Evaluación físico-química de carnes 'para hamburguesas bajas en grasa con inclusión de harina de Chinchoncho (Cajanus cajan) como extensor, Rev. Cient. Fac. Ciencias Vet. U. Zulia: 22(6), 506-512 (2012)

Gök, V., y otros tres autores, Effect of ground poppy seed as a fat replacer on meat burgers, Meat Science: 89, 400-404 (2011)

Gómez, L., O. Figueroa y J. Zapata, Actividad antioxidante de hidrolizados enzimáticos de plasma bovino obtenidos por efecto de Alcalasa® 2.4 L, Información Tecnológica: 24(1), 33-42 (2013)

Hernández, S. y N. Güemes, Efecto de la adición de harina de cascara de naranja sobre las propiedades fisicoquímicas, texturales y sensoriales de salchichas cocidas. Ciencia y tecnología de la carne: 4(1), 23 36 (2010)

INVIMA, Manual de técnicas de análisis para control de calidad microbiológica de alimentos para consumo humano, Bogotá, Colombia (1998)

Isaza, J., y otros cuatro autores, Producción y propiedades funcionales de plasma bovino hidratado en embutido tipo salchichón, Rev. Ciencias Pecuarias: 23(2), 199-206 (2010)

Julio, L., K. Atencia YP. Montero, Elaboración de un producto cárnico con incorporación de plasma sanguíneo. Rev. Ciencias e ingeniería al día U. Cartagena: 1(1), 65-69 (2011)

Julio, L., P. Montero y D. Acevedo, Plasma sanguíneo de diferentes especies: una alternativa en la industria alimentaria, Rev. Reciteia: 13(1), 37-50 (2013)

Manchimba, J. y D. Pambaquishpe, Estudio de la sustitución de tocino de cerdo por pasta de maní Arachishypogaeay la aplicación del spray dried beef plasma en la elaboración de salchicha tipo Frankfurt, Tesis pregrado, Universidad técnica del norte, Ibarra, Ecuador (2007)

NTC 1325, Instituto Colombiano de Normas Técnicas y Certificación, Establece los requisitos y las normas técnicas y certificación que contienen los métodos de ensayo que deben cumplir productos cárnicos procesados no enlatados. Quinta Actualización, Bogotá, Colombia (2008)

NTC 1662, Instituto Colombiano de Normas Técnicas y Certificación, Carne y productos cárnicos, Métodos de determinación del contenido de Grasa Total, Métodos de referencia y método de rutina, Segunda Actualización, Bogotá, Colombia (2008)

NTC 1663, Instituto Colombiano de Normas Técnicas y Certificación, Carne y productos cárnicos, Métodos de determinación del contenido de Humedad, Métodos de referencia y método de rutina, Primera Actualización, Bogotá, Colombia (2009)

Pacheco, W., D. Restrepo y J. Sepúlveda, Revisión: Uso de Ingredientes no Cárnicos como Reemplazantes de Grasa en Derivados Cárnicos, Rev. Fac. Nal. Agr. Medellín: 64(2), 6257-6264 (2011)

Rodas, A., y otros cuatro autores, Adición de plasma y paquete globular en la formulación de jamones cocidos, Rev. Cient. Fac. Ciencias Vet. U. Zulia: 8(1), 35-39 (1998)

Rodríguez, L., A. Pérez y M. Campderrós, Plasma bovino tecnológicamente mejorado para aplicaciones alimenticias, La Alimentación Latinoamericana: 93, 44-48 (2011)

Vásquez, C., S. Soto y L. Villalobos, Efecto de la fibra dietética sobre la textura de salchichas tipo Viena, Nacameh: 4(2), 37-43 (2010)

Viana, F., y otros cuatro autores, Quality of ham pâté containing bovine globin and plasma as fat replacers, Meat Science: 70(1), 153-160 (2005) 